Recherches en didactique des langues et des cultures

Les cahiers de l'Acedle

7-2| 2010

Les langues tout au long de la vie: Permanences et évolutions en didactique des langues

\title{
Préparation à l'autonomie tout au long de la vie : des cahiers de bord à l'université
}

Nathalie Gettliffe

\author{
(2) OpenEdition \\ Journals \\ Édition électronique \\ URL : http://journals.openedition.org/rdlc/2135 \\ DOI : $10.4000 /$ rdlc. 2135 \\ ISSN : 1958-5772 \\ Éditeur \\ ACEDLE
}

Référence électronique

Nathalie Gettliffe, « Préparation à l'autonomie tout au long de la vie : des cahiers de bord à l'université », Recherches en didactique des langues et des cultures [En ligne], 7-2 | 2010, mis en ligne le 01 octobre 2010, consulté le 01 mai 2019. URL : http://journals.openedition.org/rdlc/2135; DOI : 10.4000/ rdlc. 2135

Ce document a été généré automatiquement le 1 mai 2019.

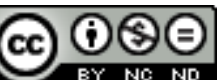

Recherches en didactique des langues et des cultures is licensed under a Creative Commons AttributionNonCommercial-NoDerivatives 4.0 International License 


\title{
Préparation à l'autonomie tout au long de la vie : des cahiers de bord à l'université
}

\author{
Nathalie Gettliffe
}

\section{Introduction}

Notre étude se situe dans le cadre d'une recherche-action telle que définie par Macaire :

... une recherche-action en didactique des langues est une intervention sur un dispositif d'enseignement / apprentissage ou de formation / accompagnement effectuée par un individu ou par un groupe, accompagné par un ou plusieurs chercheurs (Macaire, 2007 : 98).

2 En ce sens, nous proposons de présenter notre cheminement en partant de notre problématique située (contextualisée) pour déboucher sur des propositions concrètes concernant l'enseignement des langues vivantes en milieu universitaire avec des niveaux de langues hétérogènes.

\section{Problématique située de l'enseignement des langues vivantes pour non-spécialistes à l'Université Jean Monnet (Saint Etienne, 42)}

\subsection{Un enseignement des langues hétéroclite}

L'université Jean Monnet est un établissement pluridisciplinaire dispersé sur cinq campus avec, pour l'enseignement des langues, des responsables par pôles de formation. On trouvera, par exemple, un professeur agrégé ${ }^{1}$ chargé de l'enseignement de l'anglais pour la faculté de droit et un professeur agrégé chargé de tous les enseignements de langues pour l'École Saint Etienne Télécom. Chaque pôle de formation décide des objectifs à atteindre pour sa formation. À titre d'exemples, pour l'École Saint Etienne Télécom, il 
s'agira de réussir le Toeic (Test of English for International Communication) puisque la réussite de ce test est obligatoire pour obtenir son diplôme d'ingénieur; pour l'Iseag (Institut Supérieur d'Études en Administration et Gestion), le niveau B2, pouvant être validé par un Certificate of Advanced English, est exigé en fin de licence. Finalement, les heures attribuées à l'enseignement des langues est variable d'un pôle de formation à l'autre : 60 heures par an pour l'École Saint Etienne Télécom et 24 heures par an pour les deux dernières années de licence de sociologie. Au final, la contextualisation de l'enseignement des langues est de rigueur dans cet établissement et, malgré la présence d'un coordinateur pédagogique et d'un groupe de réflexion sur l'enseignement des langues vivantes, chaque responsable se "débrouille" avec les heures et les formateurs qui lui sont attribués.

\subsection{Absence de moyens}

Malgré des tentatives récentes (et plus anciennes) de coordonner l'enseignement des langues sur les campus de l'Université Jean Monnet, il n'existe pas à ce jour de centre de ressources en langues commun à toutes les formations, que ce soit en version physique (une salle regroupant des ressources en langues) ou en version numérique (un portail pointant vers diverses ressources ou activités en ligne). Le seul centre de langues existant se situe dans le bâtiment du service universitaire de formation continue et n'est accessible qu'aux personnes en activité désireuses de suivre une formation en langues étrangère.

\subsection{Des niveaux d'entrée hétérogène}

5 À l'université Jean Monnet, la plupart des enseignements de langues ne sont pas organisés par niveaux de langue ( $\mathrm{A} 1 \mathrm{à} \mathrm{C} 2 \mathrm{du}$ Cadre européen commun de référence des langues) mais selon les années d'étude. Ainsi, dans le domaine des sciences humaines et sociales, tous les étudiants de deuxième année de licence se trouvent dans le même cours quel que soit leur niveau de langue. De plus, chaque étudiant a la possibilité de choisir son enseignement de langue chaque semestre. On peut donc, avoir des étudiants qui choisissent anglais en semestre 2, puis espagnol en semestre 3 et qui reviennent en anglais en semestre 4 . En sciences humaines et sociales, la notion de progression ou de parcours en langues n'existe pas. D'ailleurs, la motivation des élèves s'en ressent puisqu'il n'existe ni prise en compte du point de départ des apprenants, ni détermination d'objectifs individuels. Avec souvent plus de quarante élèves par classe, il est difficile de concevoir une pédagogie différenciée réalisable pendant les heures de cours.

C'est donc dans ce contexte que nous avons décidé de mettre en place un dispositif plus personnalisé visant l'autonomisation des apprenants et articulé autour des "cahiers de bord ". Nous avons pensé qu'en analysant ces derniers nous serions plus à même d'avoir une lecture sur les capacités des étudiants à gérer leurs apprentissages et les modifications éventuelles à apporter au dispositif² 2 . 


\section{Mise en place d'un dispositif d'auto-formation autour du cahier de bord}

\subsection{Le cahier de bord}

\subsubsection{Historique et définition du cahier de bord}

7 La première représentation collective qu'on peut avoir du cahier de bord est liée à la notion de carnet de voyages. On pense alors au journal de voyage de Marco Polo "Le livre des merveilles" (1298), au journal de Christophe Colomb (1492), bref, aux journaux de bord des explorateurs et des colonisateurs de terres nouvelles qui revenaient au pays raconter ce qu'ils avaient observé. D'un point de vue énonciatif, ces récits sont présentés sous une forme chronologique et sont souvent accompagnés de planches de dessins. La première personne est de rigueur afin de témoigner que l'auteur a bien vu "toutes ces merveilles" de ses propres yeux. Au fil des siècles, des écrivains s'emparent de ce genre narratif pour raconter leur voyage, comme par exemple Montaigne en 1580-1581 lors de sa tournée européenne des thermes pour soigner ses calculs (son "Journal de voyage" sera publié en 1774). De nos jours, n'importe quel internaute peut partager ses impressions de voyage par le biais d'un blog (weblog).

8 A cette forme canonique (date, emploi du "je", illustrations/photos), s'ajoute une part plus ou moins grande de réflexivité par rapport à ce qui est observé. On pense notamment au cahier de l'ethnographe qui est souvent organisé avec les données (ou des transcriptions) sur la page de droite et les réflexions sur la page de gauche. Le carnet de bord tel qu'élaboré dans des centres de ressources en langue possède aussi cette partie invariante sur la réflexivité (travail effectué/appréciations personnelles). Le carnet de bord peut même ne consigner que des réflexions par rapport à des apprentissages (Macré, 2009), à des confrontations avec l'Étranger (Develotte, 2006) ou des processus de conceptions d'outils (Guichon, 2007). Cette pratique s'apparente parfois au journal intime avec une projection du soi très importante (ibid. : 11).

Pour notre part, nous avons choisi de demander aux apprenants un produit hybride qui contient de l'observable (les activités réalisées) et de la réflexivité. Étant donné que son format s'apparente le plus souvent à un cahier d'exercices, nous avons pensé qu'il serait opportun de le baptiser "cahier de bord" afin de lui donner, par la même occasion, un ancrage didactique. Nous verrons son format plus en détail dans le cadre de la description du dispositif.

Figure 1 - Typologie schématique des différents carnets de bord.

\begin{tabular}{|l|l|l|}
\hline \multicolumn{2}{|c|}{} & \multicolumn{1}{|c|}{ Niveau de réflexivité } \\
\hline $\begin{array}{l}\text { Carnet de voyages } \\
\text { Weblog }\end{array}$ & $\begin{array}{l}\text { Cahier d'ethnographe } \\
\text { Cahier de bord (didactique : activités } \\
\text { et réflexions) }\end{array}$ & $\begin{array}{l}\text { Carnet de bord pour } \\
\text { centre de langues } \\
\text { Journal d'étonnement } \\
\text { Journal intime }\end{array}$ \\
\hline Remarques & Réflexions & Introspection \\
\hline
\end{tabular}




\begin{tabular}{|c|l|c|}
\hline $\begin{array}{l}\text { Données (observations, } \\
\text { illustrations, photos) }\end{array}$ & $\begin{array}{l}\text { Données (observations, transcriptions, } \\
\text { documents bruts écrits, audio, visuels, } \\
\text { audio-visuels) }\end{array}$ & Pas de données brutes \\
\hline Date (chronologie) \\
\hline Utilisation du "je"
\end{tabular}

\subsubsection{Le cahier de bord comme outil d'individualisation de l'enseignement}

10 Comme souligné plus haut, le cahier de bord a été pensé pour une individualisation de l'enseignement dans un contexte où les niveaux de langues étaient très hétéroclites et où il n'existait pas de centre de langues. Il permet de mettre en place, en dehors de la salle de classe, une pédagogie différenciée en imposant des objectifs communs minimum. Les étudiants les plus faibles sont libres de passer autant de temps que nécessaire sur leurs points de difficultés et les meilleurs peuvent aller chercher des activités plus complexes que celles proposées.

11 Au rythme de travail imposé dans la salle de classe se substitue un rythme de travail individuel avec un choix dans les ressources proposées par l'enseignant et une liberté dans la succession des activités. Cependant, comme le rappelle Barbot (2000: 17), ce n'est pas parce que l'apprentissage est individualisé (parcours à son rythme vers des objectifs communs) qu'un apprenant devient autonome. Il existe de nombreux dispositifs qui sont présentés comme individuels mais qui restent autoritaires. Nous allons donc maintenant envisager ce qui pourrait faire du cahier de bord un outil autonomisant.

\subsubsection{Le cahier de bord comme outil d'autonomisation de l'apprenant}

12 L'autonomie a été définie dans les premiers écrits de chercheurs comme un produit final. Ainsi, Holec (1979: 4) avance qu'un apprenant autonome est un apprenant capable de prendre en charge son apprentissage, c'est-à-dire d'assumer la responsabilité de la détermination des objectifs, la définition des contenus et des progressions, la sélection des méthodes et techniques mises en œuvre, le contrôle du déroulement de l'acquisition proprement dite (rythme, moment, lieu, etc.) et l'évaluation de l'acquisition réalisée.

13 La conception actuelle de l'autonomie s'éloigne de cette vue qualifiée par certains de radicale (Allford, 2007) pour comprendre l'autonomie comme une fin et un moyen (Barbot, 2000). A cet effet, les recherches actuelles se centrent sur les différents degrés qui peuvent exister dans le processus d'autonomisation (Little, 1991 : autodétermination et contrôle), les indicateurs d'autonomie (Macré, 2009 ; Candas, 2009) ou les guidages qui peuvent être proposés (Rivens-Mompean \& Eisenbeis, 2009).

14 En tout état de cause, le cahier de bord apparaît comme un outil pour l'apprenant afin d'asseoir de manière systématique, si ce n'est automatique, un processus de réflexivité sur ces apprentissages afin qu'il détermine mieux ses objectifs, ses contenus, ses progressions, ses évaluations, etc. En résumé, toutes les composantes d'un apprentissage en autonomie ou tout du moins d'un apprentissage en semi-autonomie si l'on considère que certains objectifs étaient fixés d'avance par l'enseignant. 


\subsubsection{Le cahier de bord comme outil de recherche sur les stratégies d'apprentissage} dehors de toute référence aux réflexions portant sur l'apprentissage et il est toujours difficile selon Oxford (2003: 75) de relier, de manière cohérente, autonomie et stratégies d'apprentissage. Carré (2005: 109) note que, pour "apprendre à apprendre" dans un processus d'autonomisation, cinq séries de compétences sont à développer: les compétences cognitives, les compétences métacognitives, les compétences sociales et relationnelles, les compétences de gestion pédagogique et les compétences de navigation et de traitement de l'information. Ces compétences ressemblent étonnamment aux stratégies d'apprentissage d'Oxford (1990) appliquées au domaine des langues : stratégies cognitives, stratégies métacognitives, stratégies affectives et stratégies sociales, stratégies de mémoire et stratégies de compensation. Un cahier de bord réunissant activités et réflexions peut permettre de comprendre les stratégies d'apprentissage mises en œuvre dans le cadre d'un travail en (semi)-autonomie et, par là-même, de relier plus formellement autonomie et apprentissage.

Nous nous tournerons maintenant vers le dispositif global entourant la mise en place du cahier de bord.

\subsection{Le dispositif}

\subsubsection{Le cours}

\section{Nombre d'heures}

Notre recherche porte sur un cours d'anglais dispensé pendant le semestre 2 à un groupe d'étudiants $(n=19)$ inscrits en licence de géographie. Le nombre d'heures hebdomadaire est de 1 heure pendant 12 semaines pour un total de 12 heures d'anglais dans le semestre. Si l'on soustrait la première séance de cours qui est une séance de présentation et la dernière séance du cours pour l'examen, il s'agit véritablement d'une formation de 10 heures.

\section{Objectifs}

Les objectifs du semestre 2 sont la compréhension de textes écrits de niveau B1/B1+ et la révision de quelques notions clés de grammaire. Nous avions décidé avec le coordinateur qu'étant donné le faible nombre d'heures proposées, il valait mieux réviser et renforcer certains acquis plutôt que de présenter du matériel nouveau. Un questionnaire oral spontané proposé à d'autres étudiants de licence avait révélé que, de manière générale, les étudiants se plaignaient d'avoir des lacunes et de ne pas avoir la possibilité de les combler avant d'aborder de nouvelles notions. Leurs commentaires portaient aussi sur la fossilisation d'erreurs et sur leur peur de l'oral. C'est pour cela que nous avions décidé d'aborder la compréhension écrite comme première compétence afin de redonner confiance aux étudiants et leur permettre de "reprendre le train".

Les cours en classe portaient uniquement sur la compréhension de textes écrits avec un travail sur les différentes manières d'aborder la compréhension (travail en groupe/travail individuel, pré-compréhension, vérification d'hypothèses, écrémage, lecture détaillée, 
étude de vocabulaire, traduction, questions à trous, synonymes, antonymes, questionnaires, vrai/faux, devinettes, analyse de discours, discussions, mots de transitions...). Les textes portaient sur des thèmes particuliers (crime, tourisme, économie, médecine, drogues, politique, école) et des listes de vocabulaire étaient proposées afin que les étudiants identifient les mots qu'ils ne connaissaient pas. D'autres textes ou supports pouvaient être travaillés par les étudiants.

Pour la grammaire, toutes les notions avaient été étudiées dans des cursus préliminaires et il avait été décidé que les étudiants pouvaient retravailler seuls ces notions à l'aide d'une compilation de ressources proposées en version papier. Les notions à connaître portaient sur les articles ( $a$, the, 睤), some/any, comparatives/superlatives, present simple/ continous, past/present perfect/pluperfect, for/since/ago, questions et négations. Les ressources proposaient les notions de plusieurs manières : soit par des "test it"/"fix it" où l'étudiant faisait une évaluation diagnostique pour se situer par rapport à la notion en question, soit par la présentation de la règle de grammaire suivie d'exercices d'applications ou d'exercices de traduction. Pour chaque exercice, des corrigés étaient proposés. Les étudiants étaient invités à chercher d'autres sources que celles proposées s'ils le souhaitaient.

\section{Examen final}

21 L'examen final portait sur la compréhension d'un texte de niveau B1 (noté sur 10) et sur la traduction d'un paragraphe simple comportant tous les points de grammaire évoqués ci-dessus (noté sur 10).

\subsubsection{Le cahier de bord}

Il a été demandé aux étudiants de tenir un cahier de bord qui leur permettait de gagner des points de bonus (jusqu'à deux points). Il devait, pour chaque session, indiquer la date et la durée de la session, laisser une trace de leur travail (copie de l'exercice réalisé) et renseigner à la fin de la session les indicateurs suivants : "j'ai appris...", "je comprends mieux...", "je veux mieux comprendre...". A la fin du cahier de bord, les étudiants devaient résumer en quelques lignes les réflexions qu'ils avaient pu avoir sur le travail accompli ${ }^{3}$. Les étudiants étaient encouragés à aller chercher d'autres ressources que celles proposées par l'enseignant et à les travailler de différentes manières. Au total, une dizaine d'heures de travail était demandée pour la compréhension écrite et les notions de grammaire. Ces cahiers de bord étaient réalisés en dehors des heures de classe et étaient ensuite lus (et " notés ") par leur enseignant.

\subsubsection{Les étudiants}

Nous avons choisi d'examiner les cahiers de bords d'un groupe d'étudiants géographes ${ }^{4}$ $(\mathrm{n}=19)$ afin d'analyser de manière détaillée les productions. Ce groupe avait la particularité d'avoir le même emploi du temps pour tous ses membres car, au niveau de l'université, il n'existe qu'une seule cohorte d'étudiants géographes ${ }^{5}$. Un test de placement de fin du semestre 1 montrait des compétences grammaticales allant de 2/20 à $14 / 20$, la médiane étant de $11 / 20^{6}$. Le groupe était mixte avec 10 filles et 9 garçons. Au total pour 19 étudiants, 17 carnets de bord ont été réalisés. 


\subsection{Analyse des résultats}

24

Après avoir tenté plusieurs regroupements des données disponibles dans les cahiers de bord, il semble que la lecture des données selon la variable $\mathrm{X}=$ nombre $\mathrm{d}$ 'heures passées à réaliser le cahier de bord semble celle qui permette de mieux dégager les grandes tendances concernant le degré d'autonomie des apprenants et les stratégies d'apprentissage utilisées. Le tableau suivant récapitule le profil des trois groupes d'étudiants en termes de temps passé sur le cahier de bord

\begin{tabular}{|c|c|c|}
\hline Moins de 9 heures & Entre 9 heures et 11 heures & Plus de 11 heures \\
\hline $4\left(+2^{7}\right)$ & 7 & 6 \\
\hline
\end{tabular}

\section{Profil du groupe ayant passé moins de 9 heures sur le cahier de bord}

Sur les 6 étudiants ayant passé moins de 9 heures sur leur cahier de bord, une observation des cahiers de bord et une analyse des commentaires finaux révèlent les caractéristiques suivantes ${ }^{8}$ :

- Les commentaires finaux sont soit absents, soit ils évoquent une absence de motivation pour ce travail en autonomie :

G2.010 "En ce qui concerne le cahier de bord, je dois avouer que je ne voyais pas son utilité au début et je trouvai même ça lassant de devoir le faire, d'autant que ça ne laissait pas trop de liberté dans le travail. Mais je me suis vite rendu compte qu'il sert beaucoup, notamment à se rendre compte que j'avais oublié beaucoup de choses, la grammaire remonte à loin ! Et de choisir les textes que l'on veut étudier est plus motivant que je le pensais".

Les commentaires finaux peuvent aussi évoquer l'utilisation du cahier de bord comme outil pour la préparation au partiel :

G2.003 "Pour conclure, ce carnet de bord m'a permis de mieux me préparer au partiel d'anglais, notamment pour la partie Grammaire".

- Les sessions de travail sont aussi peu nombreuses et espacées. En douze semaines, la moyenne du nombre de session est de 4 avec des vides pouvant aller jusqu'à deux mois ;

- Les activités réalisées sont parfois corrigées avec un stylo de couleur et un marquage simple indique si la réponse est bonne ou mauvaise ;

- La mise en page est sommaire avec l'utilisation de peu de couleurs ;

- Les activités s'appuient surtout sur les ressources proposées par l'enseignant.

Un cahier de bord se différenciait des autres : un étudiant avait réalisé un travail de 13 pages en 5 heures. L'observation du contenu laissait apparaître que cet étudiant avait surtout travaillé les ressources grammaticales proposées par l'enseignant pour réviser des notions. Les deux textes écrits étudiés étaient des textes déjà travaillés en classe. Au vu de la note de l'étudiant à l'examen final (18/20), nous pouvons conclure que cet apprenant avait visiblement un niveau de langue plus élevé que la moyenne et qu'il avait donc utilisé le cahier de bord comme un outil de révision par rapport aux objectifs communs fixés par l'enseignant. Il est évident que cet étudiant n'a pas compris qu'il pouvait se fixer lui-même d'autres objectifs et dépasser les ressources proposées. 

l'examen final. Bien que nous ayons à disposition des pré-tests qui nous permettaient de connaitre le niveau des apprenants, nous n'avons pas souhaité faire l'analyse des progressions des apprenants en fonction du nombre d'heures passées sur les cahiers de bord. En effet, tous les apprentissages ne se trouvaient pas consignés dans les cahiers de bord et il était possible, comme le montre le cas de ces deux derniers étudiants, de réussir les objectifs du cours sans s'être appuyé sur un cahier de bord.

\subsubsection{Profil du groupe ayant passé plus de 11 heures sur le cahier de bord}

Pour les six étudiants ayant passé plus de 11 heures sur leur cahier de bord, les tendances suivantes se dégagent :

- Une volonté de remédier à ses difficultés ou à améliorer ses compétences :

G2.001 "Les diverses règles et exercices effectués dans le carnet de bord ont permis de revoir les bases des temps...Les bases revues et fixées ont permis de passer à un niveau supérieur de compréhension et de conjugaison" ;

G2.013 "L'ensemble des activités effectuées constituent un très bon travail de révisions" ;

- Une volonté de dépasser les objectifs du cours et se fixer ses propres objectifs (travailler pour soi) ;

- Une organisation générale pensée à l'avance avec, par exemple, des sections séparées pour les parties grammaire, textes, vocabulaire. On peut aussi parfois observer des en-têtes pour chaque session de travail avec des objectifs précisés :

G2.015 "maîtriser l'emploi et la forme des comparatifs et des superlatifs" ;

- Des fiches récapitulatives en fin de session avec un fléchage explicite des difficultés et des notions à revoir. Ces évaluations au fil des apprentissages peuvent être récapitulées dans les commentaires finaux ou dans une table des matières qui liste les points travaillés avec, à son côté, des annotations figuratives (émoticônes) (voir annexe 3 : auto-évaluation formatrice) ;

- Des séances régulières et rapprochées avec des séances plus courtes ou plus longues selon les activités choisies ;

- Un investissement dans la mise en valeur des documents et des écrits (couleur ; surlignage) (voir annexe 4) ;

- Une variété dans les activités réalisées qui dépasse le recopier/barrer/corriger. Pour certains, il s'agissait même d'un véritable défi que d'imaginer des exercices variés : G2.013 "Cette activité [compréhension écrite] m'a permis d'aborder plein de supports différents, de manière différente : recette avec acquisition d'un vocabulaire spécifique, thème d'actualité, quelques exercices de rédaction et de traduction... Pour aucun d'entre eux, j'ai réalisé la même chose" ;

- Des activités qui s'appuient sur des ressources, en partie, autres que celles proposées par l'enseignant (ex : réinvestissement de documents utilisés les années précédentes, ...);

- Une prise de conscience du décalage entre le travail effectué en classe et le travail en autonomie (voir par exemple annexe 5);

- Une identification des stratégies porteuses :

G2.015 "Les textes que j'ai analysés donc compris puis commentés m'ont entraîné à utiliser une méthode globale dans la compréhension du texte. En effet, cette méthode consiste à sélectionner les mots-clefs dans une phrase afin d'en tirer le sens principal lors d'une compréhension écrite. De plus, les commentaires de textes semblent un bon moyen pour travailler son expression". 


\subsubsection{Profil du groupe ayant passé entre 9 heures et 11 heures sur le cahier de bord} 9 heures et 11 heures sur leur cahier de bord ont adopté des comportements mixtes en reprenant des caractéristiques évoquées pour les deux groupes précédents.

\subsection{Discussions}

\subsubsection{Niveau $\mathrm{AO}$ de l'autonomie}

L'analyse des cahiers de bord d'étudiants fraîchement issus des années lycéennes nous a permis, dans un premier temps, d'identifier un niveau de départ en terme d'autonomie pour les étudiants s'étant le moins investis dans cet outil ( $<9$ heures). En s'appuyant sur la capacité à apprendre en autonomie, telle que définie par Holec (1988) :

...savoir s'y prendre pour définir CE QUE l'on va apprendre en fonction de ses besoins et/ou de l'acquisition déjà réalisée, COMMENT l'on va apprendre (choix des documents et des supports et modes d'emplois de ces supports) et COMMENT ÉVALUER les résultats atteints ainsi que la pertinence des décisions prises en ce qui concerne le QUOI et le COMMENT (Holec, $1988: 3$ ),

un niveau $\mathrm{A} 0$ peut être défini de la manière suivante pour ces étudiants.

Tout d'abord, ces étudiants ont des difficultés à situer leur niveau et, par là même, à se fixer des objectifs personnels. Dans ce cadre-là, il serait certainement intéressant de proposer des outils d'évaluation diagnostique, comme le portfolio européen des langues, afin de suggérer un ou plusieurs points de départ pour leur permettre de dépasser les propositions de l'enseignant. L'absence de commentaires intermédiaires dans leur cahier de bord montre aussi qu'il leur est difficile de se projeter et d'établir une progression dans leurs apprentissages. Il serait peut-être intéressant, en classe, de revenir sur l'utilité des commentaires intermédiaires afin de différencier les acquis (j'ai appris..., je comprends mieux...) des nouveaux objectifs éventuels (je veux mieux comprendre...).

Concernant le COMMENT, ces étudiants possèdent quelques techniques de base pour ce qui est du travail sur des supports proposés par l'enseignant (barrer/corriger) bien que ces dernières relèvent, en fait, d'un transfert de techniques utilisées par cet enseignant dans un cadre traditionnel (utilisation du stylo rouge et des annotations pédagogiques juste/faux). Ils sont aussi capables d'organiser des sessions de travail bien qu'elles soient irrégulières et qu'elles relèvent, elles aussi, d'une conception classique du travail (type devoir à effectuer à la maison).

Enfin, l'ÉVALUATION pratiquée par ces étudiants reste minime puisque d'une part les étudiants évaluent peu leur travail (absence de commentaires finaux) et que, d'autre part, ils ne remettent pas en cause le/leur choix des supports ou la/leur définition des objectifs/contenus. Il faudrait certainement proposer un travail réflexif sur l'importance des boucles récursives entre le travail planifié, le travail effectué, une auto-évaluation de ce dernier et un réajustement du QUOI et du COMMENT. Á ce titre, les schémas sur l'autorégulation tels que proposés par Zimmerman (2000) pourraient permettre aux élèves de visualiser les processus à mettre en place. 


\subsubsection{Indicateurs d'autonomie et échelles}

Comme le note Benson (2001: 50-51), le concept d'autonomie est une notion à plusieurs facettes qui peut reposer sur les trois piliers de l'auto-régulation : réguler la gestion de ses apprentissages, réguler les processus d'apprentissage et réguler les situations d'apprentissage. Dans ce contexte multiforme, il est donc difficile d'établir des gradations entre un niveau de départ et une "autonomie totale", d'autant plus que, comme pour chaque apprentissage, il existe des périodes de régression. Cependant, bien que mesurer le degré d'autonomie d'un apprenant reste une tâche complexe, l'analyse des cahiers de bord permet de pointer vers des indicateurs d'autonomie qui peuvent être agencés sur des échelles et permettre éventuellement la mise en place de processus de guidage pour le développement de l'apprenance (Carré, 2005).

Le premier indicateur que nous avons relevé concerne la capacité et/ou la volonté de réfléchir sur son parcours (Macré, 2009) à des degrés divers. Ceci est exemplifié par la présence ou l'absence de commentaires finaux ou intermédiaires. Nous distinguons capacité et volonté car l'apprenant peut avoir les capacités d'apprendre en autonomie mais peut ne pas vouloir le montrer (Holec, 1979) ou il peut ne pas être motivé pour se prendre en main (Littlewood, 1996: 428). Citons le cas des étudiants qui affichent franchement leur manque de motivation, qui affirment ne pas percevoir l'intérêt du cahier de bord ou l'utilisent simplement pour réviser dans le cadre des examens en dehors de toute réflexion sur leurs apprentissages.

Un second indicateur concerne la capacité à s'organiser dans le temps. Ceci est illustré par la régularité ou l'irrégularité des sessions de travail pouvant même aller jusqu'au décrochage (Blin, 1998).

Un troisième indicateur porte sur la capacité à trouver des supports pour son apprentissage. On observe un phénomène de dépendance (op.cit) pour les moins autonomes qui s'accrochent au matériel proposé par l'enseignant alors que les plus performants n'hésitent pas à explorer (Candas, 2009) d'autres voies même si ce n'est pas toujours en adéquation avec leurs objectifs.

Un quatrième indicateur mis en exergue par les cahiers de bord touche à la capacité à gérer ses angoisses face à ses auto-apprentissages. Les commentaires finaux font état d'étudiants expliquant leurs moments de découragements ou leur prise de confiance face à la résolution de difficultés. Rivens-Mompean et Eisenbeis (2009) avaient déjà mis en avant la nécessité de développer cette capacité par des procédures de guidage.

Au total, les indicateurs d'autonomie dégagés peuvent permettre dans un second temps d'élaborer des séances de mises en commun sur l'apprendre à apprendre ou de mettre en place des procédures de guidage (rencontres avec un conseiller, évaluation réflexive, ...). Ceci permettrait aux apprenants les moins autonomes de prendre plus en charge leurs apprentissages et de se situer sur le cheminement vers l'autonomie tout au long de la vie. Pour le reste des apprenants, nous avons noté dans l'analyse des cahiers de bord que les formats de travail de départ n'avaient quasiment pas évolué en l'espace d'une dizaine d'heures alors que dans d'autres circonstances (voir séances-conseil, Candas, 2009), les étudiants étaient capables de dépasser leurs représentations initiales de l'autonomie et de mettre en place des routines autonomisantes. Il serait, de nouveau, opportun de faire collaborer les étudiants afin qu'ils élaborent un plan d'autonomisation en langues qui pourrait reposer sur des critères autres que ceux extraits de notre analyse ci-dessus (par 
exemple, profils d'apprenants autonomes, Tremblay, 2003, Candy, 1991; niveaux d'autonomie, Blin, 1998).

\subsubsection{Stratégies observées}

41 Comme nous l'avions indiqué dans la présentation de notre étude, le cahier de bord est aussi un outil de recherche qui permet, par le biais, de la consignation des activités réalisées, de faire état des différentes stratégies utilisées en autonomie. En regroupant les catégories d'Oxford (1990) et de Carré (2000), les stratégies observées dans les cahiers de bord sont les suivantes :

- Mémorielle (utiliser des mot-clés);

- Cognitive (répéter/ réécrire, reconnaître des éléments qui se répètent, déduire, analyser des expressions, traduire, résumer, surligner) ;

- Métacognitive (lier les apprentissages à des données antérieures, (s') organiser : définir des buts et des objectifs, s'évaluer) ;

- Affective (s'encourager, prendre des risques, utiliser un journal de bord) ;

- Sociale (demander des clarifications, demander des corrections);

- Gestion pédagogique (choisir le rythme et la durée des sessions);

- Navigation (localiser le contenu).

Selon Benson (2000), la capacité à réguler la gestion de ses apprentissages (control over learning management) repose essentiellement sur la capacité à comprendre les stratégies d'apprentissage disponibles pour l'étudiant et évaluer celles qui sont les plus efficaces pour des buts précis. Il est évident que, dans notre cas, les stratégies utilisées sont largement individuelles puisque centrées sur un produit individuel et que notre dispositif gagnerait à s'appuyer sur des mises en commun pour permettre une meilleure mise en œuvre des stratégies affectives et sociales si nécessaires dans le cadre de la communication langagière.

\section{5. Évaluation du dispositif}

En examinant des discussions proposées ci-dessus, il convient de revenir tout d'abord sur la nécessité de prendre en compte le faible niveau d'autonomie de certains étudiants (au moins un tiers des apprenants dans le cadre de notre étude). Bien que le cahier de bord ait été balisé pour permettre la mise en place des comportements visant l'autonomisation des apprenants, certains étudiants ne sont pas en mesure de saisir les enjeux d'un travail en autonomie. Un travail sur le concept même de l'autonomie, proposé par Barbot (2000 : 20) "Pour vous, l'autonomie c'est quoi ?", pourrait permettre de dépasser "l'empilement d'exercices sans réelle réflexion sur les apprentissages" tel que pratiqué lors de la reconduction du dispositif pendant une seconde année.

Cependant, pour cela, comme le note un responsable de formation, il faudrait non seulement faire comprendre l'intérêt du travail en autonomie aux apprenants mais aussi former et convaincre les enseignants du bien-fondé d'un tel dispositif: "C'est autant la 
formation de l'enseignant que de l'étudiant qui est en cause". Nous nous situons donc au cœur du problème si nous souhaitons donner une signification à notre recherche-action.

Notre analyse des données et nos discussions permettent d'avancer : il est impératif d'inscrire le cahier de bord dans un dispositif d'autonomisation plus large. En effet, en dehors de la prise en charge des moins autonomes, notre dispositif initial devrait :

- s'appuyer sur des enseignants formés à l'apprentissage des langues par l'auto-formation ;

- sensibiliser les étudiants aux contraintes de l'apprentissage en milieu institutionnel universitaire (examens communs, objectifs imposés...) afin qu'ils pensent l'autonomie plus dans une logique d'autonomie réactive que proactive (Littlewood, 1999);:

- présenter et décliner les processus de réflexivité car ils font partie intégrante du développement de l'autonomie, notamment en terme de déconditionnement (Holec, 1980) par rapport à des manières d'apprendre "classique" et pour mieux comprendre les processus mentaux impliqués dans l'apprentissage d'une langue étrangère. Cet habitus réflexif pourrait ensuite être transposé dans d'autres contextes d'apprentissage tout au long de la vie ou même servir à conduire des recherches (de Robillard, 2009) ;

- présenter et décliner les stratégies d'apprentissage qui permettent aux étudiants de mieux gérer leurs auto-apprentissages. Des séances communes telles que proposées dans le dispositif de Macré (2009) pourraient aussi permettre de travailler plus en profondeur les stratégies liées à l'affectif et au social ;

- proposer des ressources ou des typologies de ressources afin que les étudiants s'investissent sur d'autres supports ;

- proposer des retours sur les cahiers de bord lors de séances individuelles (type séanceconseil) ou pendant les cours (travail par groupe ou debriefing commun).

\section{Conclusion}

Notre recherche-action a pu démontrer le bien-fondé de l'introduction d'un cahier de bord consignant activités et réflexions dans un dispositif d'enseignement des langues en milieu institutionnel avec des niveaux de langues hétérogènes. En effet, plus des $2 / 3$ des étudiants se sont emparés de cet outil pour un réel travail en autonomie. Cependant, en dehors des avancées sur les stratégies utilisées par ces derniers ou sur les degrés d'autonomisation existants, notre dispositif gagnerait à dépasser la simple introduction de cet outil pour déployer un véritable arsenal de démarches en vue de l'autonomisation, incluant notamment une formation plus poussée des enseignants aux concepts de l'autoapprentissage et en invitant les cahiers de bord dans la salle de classe pour dépasser les pratiques initiales.

\section{BIBLIOGRAPHIE}

Allford, D. \& Pachler, N. (2007). Language, Autonomy and the New Learning Environments. Oxford: Peter Lang.

Barbot, M.-J. (2000). Les auto-apprentissages. Paris : CLÉ international. 
Benson, Ph. (2001). Teaching and Researching Autonomy in Language Learning. Harlow: Pearson Education.

Blin, F. (1998). "Les enjeux d'une formation autonomisante de l'apprenant en environnement multimédia". In Chanier, T., Pothier, M. (dirs), "Hypermédia et apprentissage des langues", Études de linguistique appliquée (éla). Vol. 110. pp. 215-226.

Candas, P. (2009). Analyse de pratiques d'étudiants dans un Centre de Ressources de Langues : indicateurs d'autonomie dans l'apprentissage. Thèse de doctorat.

Candy, P. (1991). Self-direction for Lifelong Learning. San Francisco: Jossey-Bass.

Carré, Ph. (2005). L'apprenance. Paris : Dunod.

Develotte, C. (2006). "Le journal d'étonnement". Lidil, vol. 34. pp.105-124.

Guichon, N. (2007). "Récits de soi en formation - L'écriture réflexive dans la formation didactique des enseignants". Actes du colloque "Le biographique, la réflexivité et les temporalités - Articuler langues, cultures et formation". Université de Tours. http://pagesperso-orange.fr/nicolas.guichon/topic4/ guichon.2007.r-flexivit-.pdf

Holec, H. (1979). Autonomie et apprentissage des langues étrangères. Strasbourg : Conseil de l'Europe. Holec, H. (1988) (dir.). Autonomy and Self-directed Learning : Present Fields of Application. Strasbourg : Council of Europe.

Little, D. (1991). Learner Autonomy. 1: Definitions, Issues and Problems. Dublin: Authentik.

Littlewood, W. (1996). Autonomy: an anatomy and a framework. System. Vol. 24, nº 4. pp. 427-35.

Macaire, D. (2007). "Didactique des langues et recherche-action". Les Cahiers de l'Acedle, vol. 4. pp. 97-120.

Macré, N. (2009). "L'analyse d'un dispositif de langues à travers des "carnets de bord" des apprenants de niveau A2 ". Les Cahiers de l'Acedle, vol. 6, nº 1. pp. 103-114.

Oxford, R. (1990). Language Learning Strategies: what every Teacher should Know. Rowley: Newbury House.

Oxford, R. (2003). "Language learning styles and strategies: An overview". TESOL Quaterly, vol, 37, $\mathrm{n}^{\circ} 2 . \mathrm{pp} .41-47$.

Rivens-Mompean, A. \& Eisenbeis, M. (2009). "Autoformation en langues: quel guidage pour l'autonomisation?" Les Cahiers de l'Acedle. Vol. 6, n ${ }^{\circ \circ} 1$. pp. 221-244.

Robillard, D. de (2009) (dir). Réflexivité, herméneutique : vers un paradigme de recherche? Rennes : Presses universitaires de Rennes.

Tremblay, N. (2003). "L'autoformation: pour apprendre autrement". Montréal : Les presses de l'université de Montréal.

Zimmerman, B. (2000). Apprenants autonomes. Bruxelles, de Boeck.

\section{ANNEXES}

\section{Annexe 1}

Quelques exemples de commentaires autoréflexifs et un exemple de conclusion 

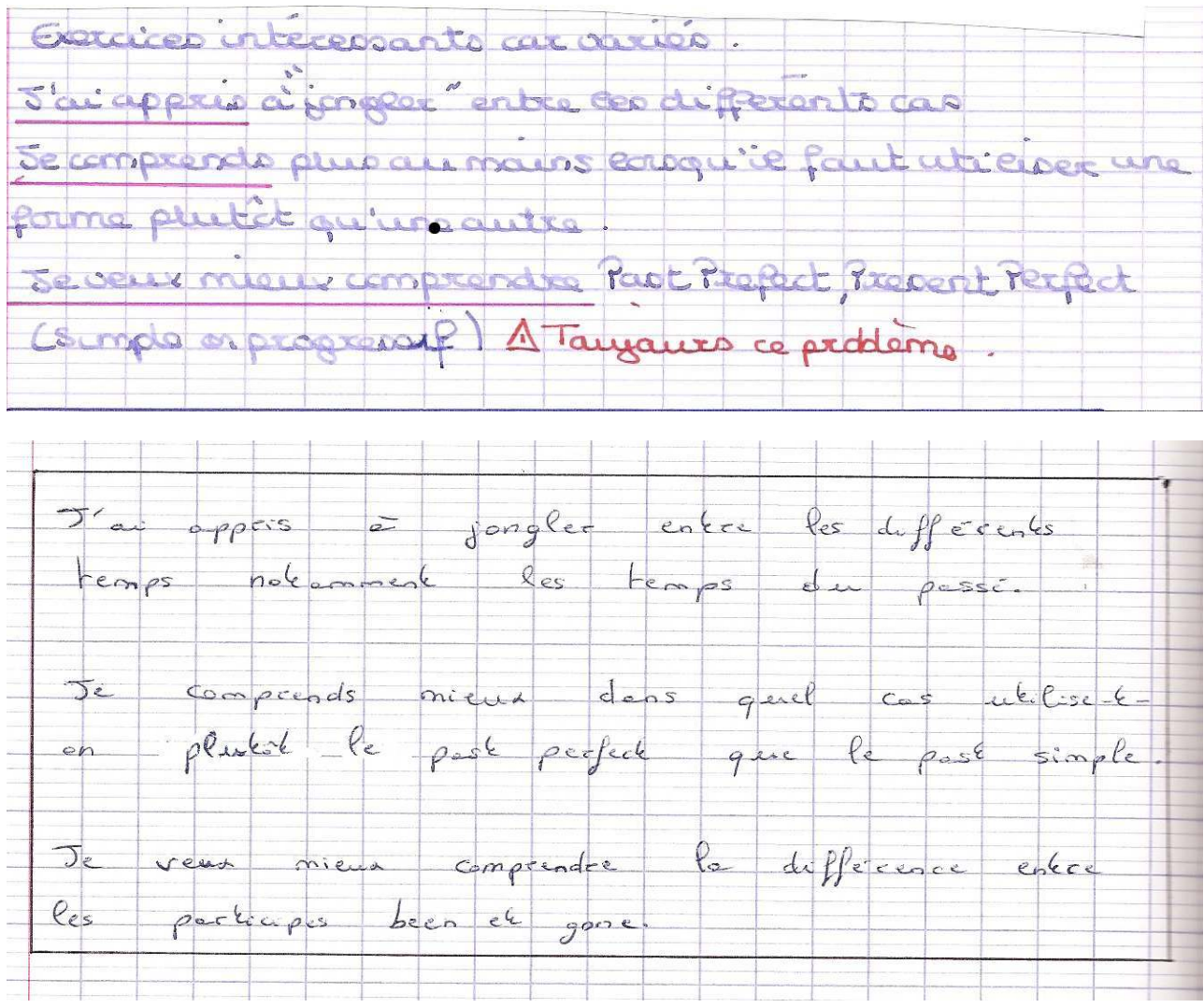

Date: $5 / 04 / 2008 \quad \frac{\text { Temps passe: }: 30 \mathrm{~min}}{\mathrm{~m}}$ Jálappris: On utilise gri" pour une pèriode, une durée contrainement à since"

on emproi pas "do" pour des questions donk le sujet eat= Who, what ow which

Pour l'inzemonëgation, on utilise la structune aux $+n^{\prime}=+$ sujel $=$ iverbe...? 


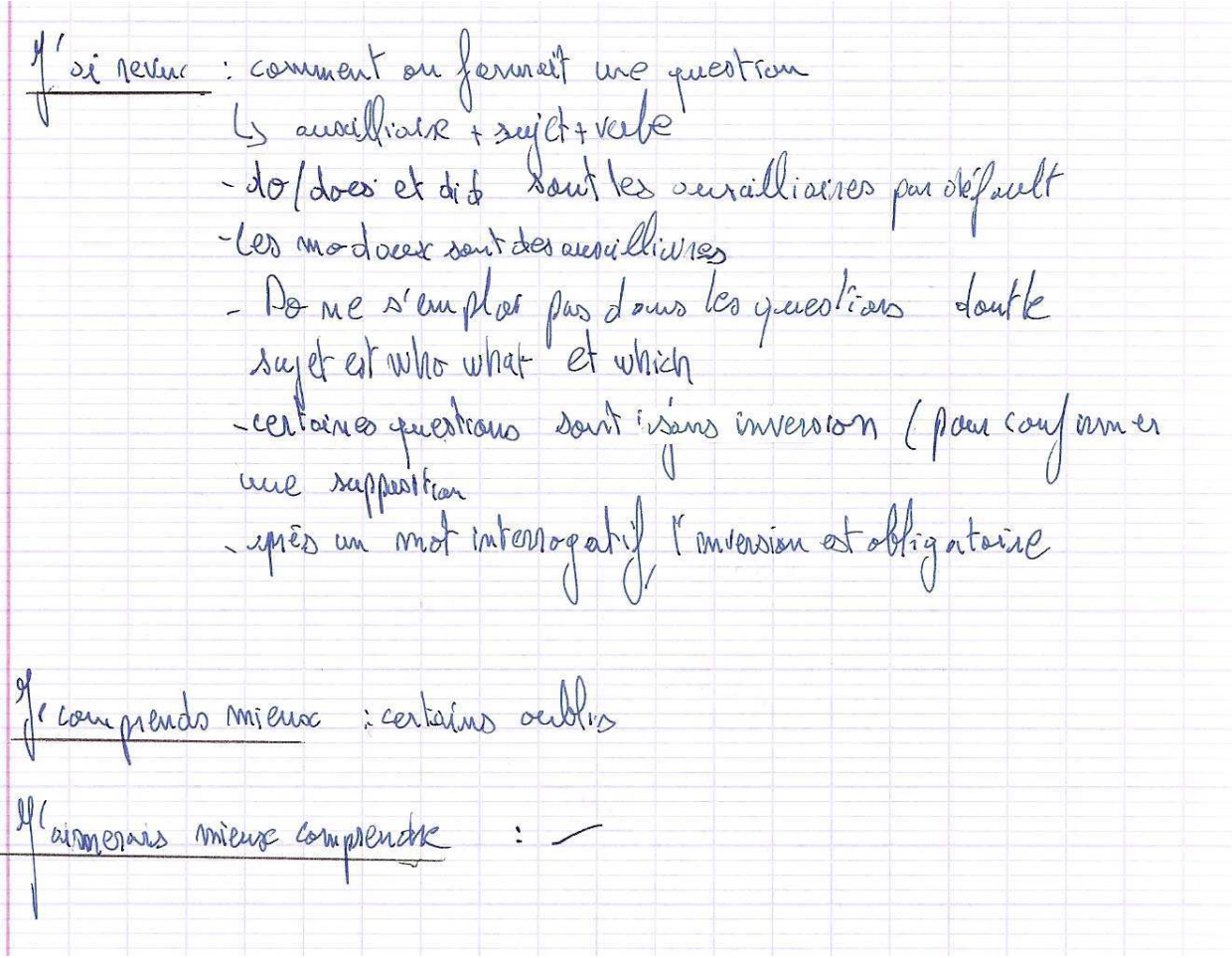

deo 12 haures d'anglais de l'annde n'étraient, bien antendu, pas suffisontes pour maintenir $l$ niveau : - Esis. En revanche, ce fut une bonne idée de oréer un cahier d'exercices où nous pouvions travailler individuelement certains points à revoir ou à apprendre, soit à l'aide de fiches a'exercices, soit à l'aide de documents choisis por rous mômes. J'ai apprécié la fait de pouvaì s'exercer sur Pes artides que l' on voulait: c'est certainement la meilleuse fagon de progresser dans une langue. Une manière ludique pour travailler!

\section{Annexe 2}




\section{Exemple d'un cahier de bord représentatif d'étudiants ayant passé moins de 9 heures à le réaliser}



\section{Annexe 3}

Table des matières avec smileys (une pratique de l'auto-évaluation formatrice)

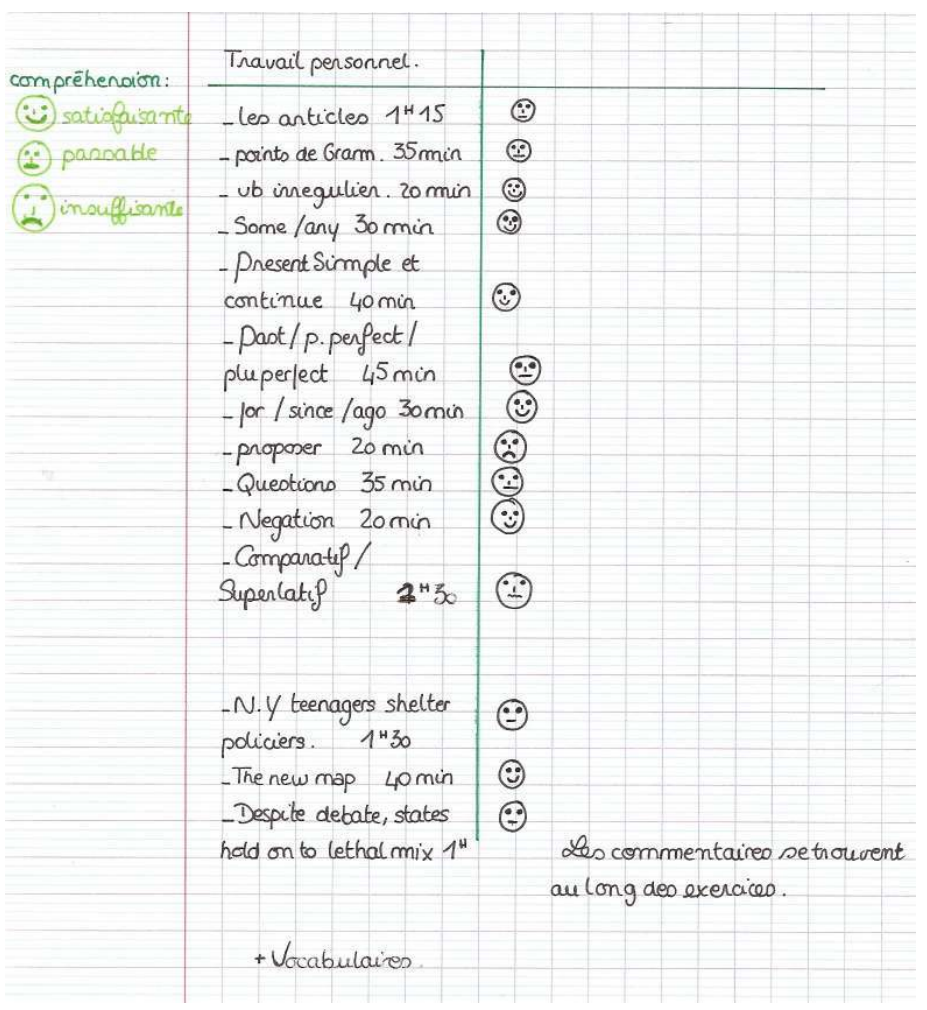


Annexe 4

\section{Mise en valeur des écrits}

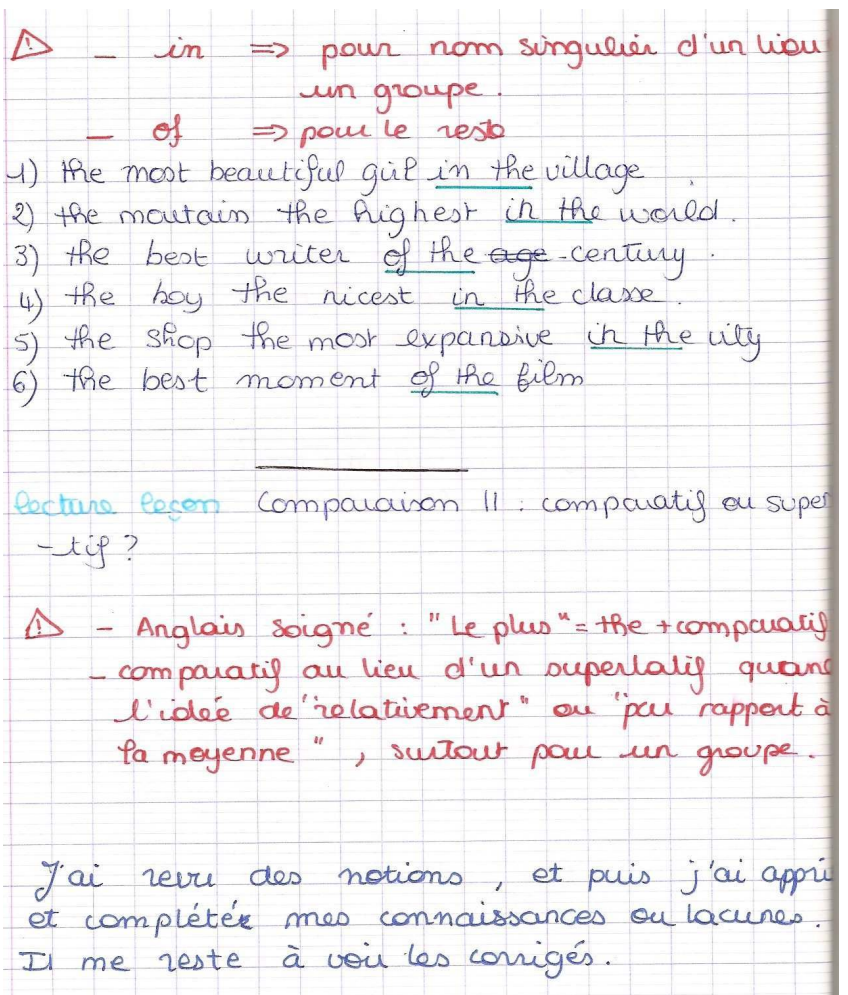

Annexe 5 
Carte collée sur un cahier de bord pour illustrer le décalage entre le travail effectué en classe (papillonage et bruitage) et le travail en autonomie (concentration)

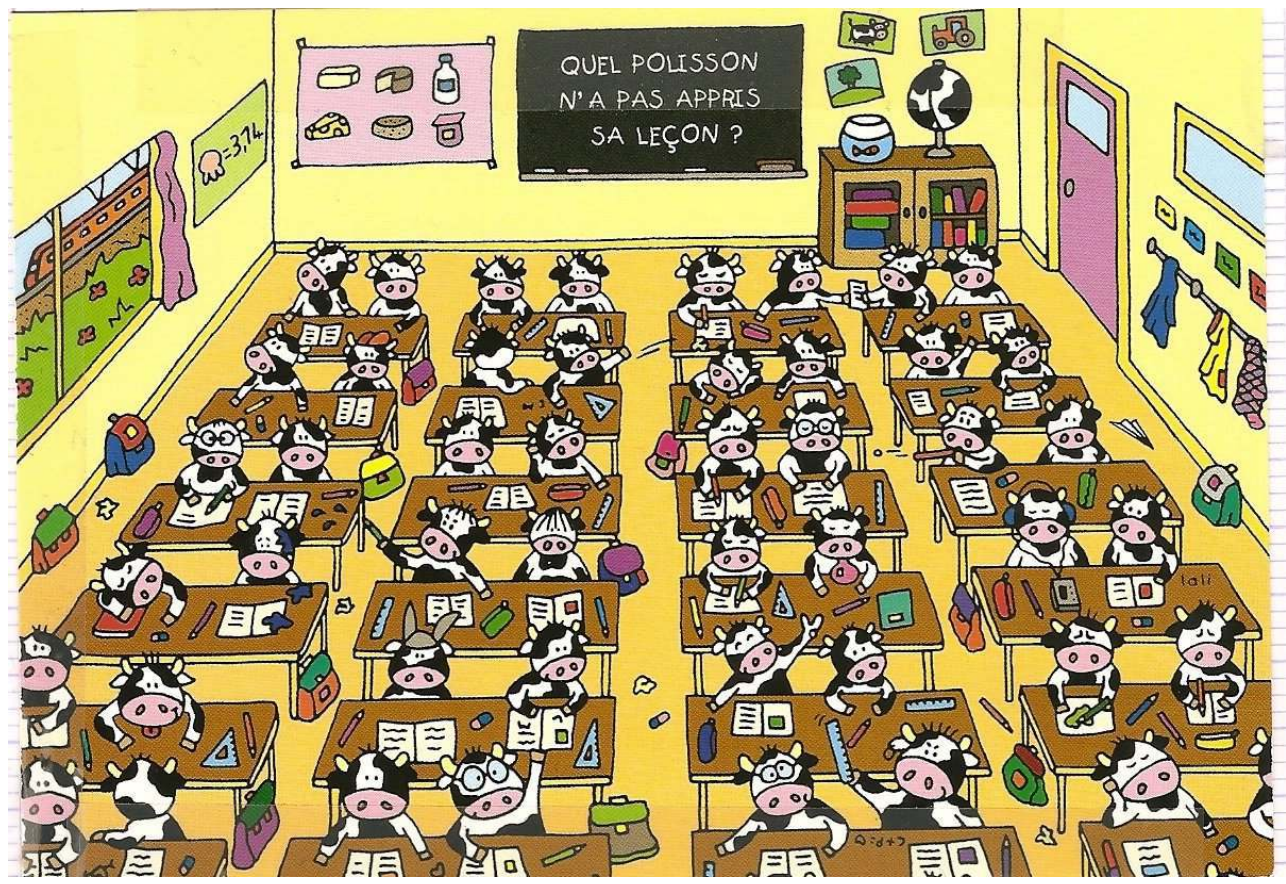

\section{NOTES}

1. Professeur agrégé = PRAG; enseignant ayant réussi le concours de l'agrégation en France.

2. Au niveau de la Vice-présidence de l'université Jean Monnet, une réflexion était engagée pour améliorer la qualité de l'offre en langues vivantes. Notre expérience pouvait aussi apporter quelques éléments de réponses.

3. Pour des exemples, voir annexe 1

4. Au total, ce sont plus de cent étudiants en sociologie, en histoire et en géographie qui ont participé de manière "volontaire"» à ce dispositif : en effet, le cahier de bord était un outil qui permettait de recevoir des points supplémentaires et de se préparer à l'examen final; ne pas le réaliser n'exposait pas l'étudiant à des pénalités.

5. Les étudiants en sociologie et en histoire étant très nombreux, les emplois du temps sont organisés avec des sous-groupes qui pouvaient ajouter une variable dans notre étude, c'est-à-dire que des étudiants présents dans un même créneau d'anglais pouvaient, en dehors de cette heure d'enseignement, avoir des emplois du temps différents, et donc une disponibilité plus ou moins fluctuante pour la rédaction du cahier de bord. .

6. Pour indication, les autres groupes de sociologues et historiens des semestres S2 avaient une valeur médiane de 7.5/20, ce qui place les étudiants géographes à un niveau supérieur.

7. Ce sont les deux étudiants n'ayant pas réalisé de cahiers de bord

8. Pour un exemple d'une page représentative des cahiers de bord de ces étudiants, voir annexe 2

9. Commentaires d'un responsable de formation 


\section{RÉSUMÉS}

Cette recherche-action vise à dégager les pratiques étudiantes et les niveaux d'autonomisation des apprenants dans la mise en place d'un dispositif autonomisant centré sur l'utilisation d'un cahier de bord en contexte universitaire avec des niveaux de langue hétérogènes au sein d'un même cours. L'analyse de cet outil (incluant activités et réflexions sur les apprentissages) a permis non seulement d'identifier un niveau A0 en autonomie pour les étudiants de première année de licence en géographie mais aussi de répertorier les stratégies utilisées par les étudiants. Une modification du dispositif initial est proposée afin de créer un contexte d'autonomisation plus large.

This action-research project investigates learner practices levels of autonomy of university students using a logbook to record activities and reflections on learning English in a multi-level language classroom. Analyses of logbooks revealed that some first-year students had very little ability to work autonomously but that others relied on a wide range of language learning strategies to accomplish their language goals. Overall, the language program should be adjusted to further the efficiency of the logbook as a component for developing autonomy.

\section{INDEX}

Mots-clés : cahier de bord, autonomie, processus d'autonomisation, indicateurs d'autonomisation

Keywords : log book, autonomy, acquisition of autonomy, autonomy competencies

\section{AUTEUR}

\section{NATHALIE GETTLIFFE}

Nathalie Gettliffe est maître de conférences au département de Linguistique appliquée et de didactique des langues de l'Université de Strasbourg et membre de l'unité de recherche EA 1339 Linguistique, Langue, Parole. Ses recherches portent principalement sur l'utilisation des technologies de l'information et de la communication en classe de langue étrangère (anglais et FLE). Elle intervient dans le Master 2 Conseiller Formateur Multilingue dirigé par Nicole Poteaux. Courriel : ngettliffe[at]unistra.fr Toile : http://prismelangues.u-strasbg.fr/

Adresse : Le Patio, 22, rue René Descartes, 67000 Strasbourg, France. 